\title{
Recent progress on the calculation of three-loop heavy flavor Wilson coefficients in deep-inelastic scattering
}

\author{
J. Ablinger ${ }^{a}$, A. Behring ${ }^{b}$, J. Blümlein ${ }^{b}$, A. De Freitas ${ }^{*}{ }^{\dagger}$ A. Hasselhuhn ${ }^{a}$, \\ A. von Manteuffel ${ }^{c}$, C. Raab ${ }^{b}$, M. Round ${ }^{b}$, C. Schneider ${ }^{a}$, and F. Wißbrock ${ }^{a, b, d}$ \\ ${ }^{a}$ Research Institute for Symbolic Computation (RISC), Johannes Kepler University, \\ Altenbergerstraße 69, A-4040, Linz, Austria. \\ ${ }^{b}$ Deutsches Elektronen-Synchrotron, DESY, \\ Platanenallee 6, D-15738 Zeuthen, Germany. \\ ${ }^{c}$ PRISMA Cluster of Excellence, Institute of Physics, J. Gutenberg University, \\ D-55099 Mainz, Germany. \\ ${ }^{d}$ Institut des Hautes Études Scientifiques, IHES, Route de Chartres 35, \\ F-91440 Bures-sur-Yvette, France \\ E-mails: jablinge@risc.uni-linz.ac.at, Arnd.Behring@desy.de, \\ Johannes.Bluemlein@desy.de, abilio.de.freitas@desy.de, \\ alexander.hasselhuhn@desy.de, manteuffel@uni-mainz.de, \\ clemens.raab@desy. de, mark . round@desy . de, \\ cschneiderisc.uni-linz.ac.at, fabian.wissbrock@desy.de
}

\begin{abstract}
We report on our latest results in the calculation of the three-loop heavy flavor contributions to the Wilson coefficients in deep-inelastic scattering in the asymptotic region $Q^{2} \gg m^{2}$. We discuss the different methods used to compute the required operator matrix elements and the corresponding Feynman integrals. These methods very recently allowed us to obtain a series of new operator matrix elements and Wilson coefficients like the flavor non-singlet and pure singlet Wilson coefficients.
\end{abstract}

Loops and Legs in Quantum Field Theory - LL 2014,

27 April - 2 May 2014

Weimar, Germany

\footnotetext{
* Speaker.

${ }^{\dagger}$ We would like to thank M. Steinhauser for the possibility to use the code MATAD. This work was supported in part by DFG Sonderforschungsbereich Transregio 9, Computergestützte Theoretische Teilchenphysik, Studienstiftung des Deutschen Volkes, the Austrian Science Fund (FWF) grants P20347-N18 and SFB F50 (F5009-N15), the European Commission through contract PITN-GA-2010-264564 (LHCPhenoNet) and PITN-GA-2012-316704 (HIGGSTOOLS), by the Research Center "Elementary Forces and Mathematical Foundations (EMG)" of J. Gutenberg University Mainz and DFG, and by FP7 ERC Starting Grant 257638 PAGAP.
} 


\section{Introduction}

In the large $Q^{2}$ limit, the heavy flavor Wilson coefficients in deep-inelastic scattering (DIS) are known to factorize into light flavor Wilson coefficients and massive operator matrix elements [1,2]. These heavy flavor coefficients can then be convoluted with parton distribution functions (PDFs) to obtain the heavy flavor corrections to deep-inelastic scattering structure functions, which amount to sizeable contributions, in particular in the region of small values of the Bjorken variable $x$. At NNLO, the light flavor Wilson coefficients are known [3]. The missing ingredients required to obtain the heavy flavor Wilson coefficients are therefore the 3-loop massive operator matrix elements (OMEs).

Here we present our latest results in the ongoing effort to calculate these quantities. Particularly, six out of eight OMEs are by now available. The simplest operator matrix elements, $A_{q q, Q}^{(3), \mathrm{PS}}$ and $A_{q g, Q}^{(3)}$, were obtained in [4]. More recently, $A_{g q}^{(3)}[5], A_{q q}^{(3), \mathrm{NS}, \mathrm{TR}}[6]$ and $A_{Q q}^{(3), \mathrm{PS}}$ [7] were calculated using a variety of techniques to be described in the following sections. Using these operator matrix elements, we have been able to obtain the Wilson coefficients $L_{q,(2, L)}^{\mathrm{PS}}, L_{g,(2, L)}^{\mathrm{S}}[8]$, $L_{q,(2, L)}^{\mathrm{NS}}[6]$ and $H_{q,(2, L)}^{\mathrm{PS}}$ [7]. The asymptotic heavy flavor corrections to $F_{L}\left(x, Q^{2}\right)$ have been calculated in Refs. [8,9]. Also, results for the terms proportional to $T_{F}^{2}$ in $A_{g g, Q}^{(3)}[10]$ and all $N_{F} T_{F}^{2}$ terms $[4,11,12]$ have been computed. Once the last two operator matrix elements are completed, and the corresponding heavy flavor contributions to the DIS structure functions are then obtained, it will be possible to make more precise determinations of $\alpha_{s}$ and the mass of the charm quark $m_{c}$, as well as provide better constraints on sea quarks and the gluon and thus improve the results given in [13-15]. The 3-loop OMEs are also needed to obtain the matching relations at NNLO in the variable flavor number scheme (VFNS) [2,16]. Starting with 3-loop order there are also heavy flavor contributions due to graphs containing massive fermion lines of different mass, see Refs. [17-19] and the talk by F. Wißbrock [20] presented at this conference. Furthermore, the asymptotic heavy flavor corrections for the charged current processes have also been calculated to next-to-leading order (NLO) [21,22].

The operator matrix elements have been calculated using the standard Feynman rules of QCD together with the Feynman rules for operator insertions as described in Refs. [6, 23]. Feynman diagrams were generated based on these rules using QGRAF [24]. The output of QGRAF was then processed using Form [25], after which the diagrams end up being expressed as a linear combination of a large number of scalar integrals. These scalar integrals are then reduced to a much smaller set of master integrals using integration by parts identities, as described in Section 2.1. The master integrals are then calculated using a variety of techniques. These will be discussed in Section 2.2, where we will make special emphasis on the differential equations method. We will discuss our results in Section 3. The conclusions are given in Section 4.

\section{Calculation of the operator matrix elements}

\subsection{Integration by parts identities}

We calculate the operator matrix elements as functions of the Mellin variable $N$, and perform the 
reduction to master integrals using the $\mathrm{C}++$ program Reduze2 $[26]^{1}$, which implements Laporta's algorithm [29]. It is not so straightforward to apply this algorithm to the case where we have operator insertions, precisely because of the dependence on the arbitrary parameter $N$. Laporta's algorithm is designed to work with integrals with definite indexing, and although it may be possible to adapt this algorithm to the case where we have an arbitrary index $N$, we found a more elegant solution by rewriting the operator insertions in terms of propagators, which will be raised to definite powers. For example, in the case of an insertion on a line, the operator insertion will be proportional to $(\Delta \cdot k)^{N-1}$, where $k$ is the momentum going through the line, and $\Delta$ is a light-like vector. We now introduce a new variable $x$ and re-express the operator insertion by the following generating function

$$
(\Delta . k)^{N-1} \rightarrow \sum_{N=1}^{\infty} x^{N-1}(\Delta . k)^{N-1}=\frac{1}{1-x \Delta . k} .
$$

In the case of 3-, 4- and 5-point operator insertions, we can similarly re-express the operator insertion in terms of products of the same type of artificial propagators. These new propagators can be added to the list of propagators defining the integrals, and Laporta's algorithm can then be applied without problems. Propagators like the one given in Eq. (2.1) are known as bilinear propagators, and Reduze 2 has been adapted to be able to deal with such objects. Auxiliary propagators are introduced when needed in such a way that all products of internal momenta with an external/internal momentum or with $\Delta$ can be uniquely expressed as a linear combination of inverse propagators. A set of propagators that satisfies this condition is called an integral family. All integrals involved in a given problem will be identified by specifying an integral family and the powers of the propagators, which can be negative if the integral has irreducible numerators. We have found that all integrals required for the calculation of all of the eight OMEs can be specified using 24 integral families.

\subsection{Calculation of the master integrals}

For the calculation of the master integrals we used a combination of one or more of the following methods, depending on the complexity of the integral under consideration:

- Summation methods, implemented in the Mat hemat i ca package Sigma [30,31], based on advanced symbolic summation algorithms in the setting of difference fields [32-40], and the packages HarmonicSums [41-44], EvaluateMulti- Sums, and SumProduction [45].

- Hypergeometric functions [47-49].

- Mellin-Barnes representations [50-53].

- In the case of convergent massive 3-loop Feynman integrals, they can be performed in terms of hyperlogarithms, generalizing the method proposed in [54] to massive diagrams with operator insertions $[49,55]$.

- Differential (difference) equations [56].

\footnotetext{
${ }^{1}$ The program Reduze2 uses the codes Fermat [27] and GiNaC [28].
} 
The summation methods and other mathematical methods we used in the representation and reduction of nested sums and iterated integrals we have used were described in detail in the talks by C. Schneider [57], J. Ablinger [58] and C. Raab [59,60] given at this conference. Some of the integrals can be completely solved in terms of hypergeometric functions (including Appell hypergeometric functions) with parameters depending on $N$ and the dimension $D=4+\varepsilon$, or multiple sums of such functions where the summation indices also appear in the parameters of the hypergeometric function. If the corresponding series representation is convergent, the resulting sums can then be performed using Sigma. A survey on the function spaces, which have appeared in the present calculations, is given in Ref. [61].

In some cases, after Feynman parameterization of the integrals, the Feynman parameters can be integrated in terms of Beta functions by splitting a denominator using $[52,53]$

$$
\frac{1}{(A+B)^{v}}=\frac{1}{2 \pi i} \int_{-i \infty}^{+i \infty} d \sigma \frac{\Gamma(-\sigma) \Gamma(\sigma+v)}{\Gamma(v)} \frac{A^{\sigma}}{B^{\sigma+v}} .
$$

The remaining contour integral in $\sigma$ can then be done with the help of the Mathemat ica package MB [62], which finds a contour and a value of $\varepsilon$ such that the Feynman integral is well defined, and then analytically continues to $\varepsilon \rightarrow 0$. After this, we can take residues and then sum them using Sigma.

The method of hyperlogarithms and its generalizations have been described in detail in [55], where a few examples were presented. This method applies to Feynman integrals which are nonsingular in the dimensional parameter $\varepsilon$. It relies on the $\alpha$-parameterization of the integrals, integrating each parameter one after the other. A required condition for the applicability of this method is that after each integration, the denominators of the integrals remain linearly factorizable in the $\alpha$ parameters. Many of the most interesting integrals appearing in our calculations do not satisfy this condition. It can be applied, if e.g. quadratic forms of Feynman parameters can be transformed away or mapped into the argument of the iterated integral. In the massive case, however, this is not always possible, whatever order of integrations is applied.

Many of the most complicated integrals we have encountered so far were solved using the differential equations method. The idea behind this method is to take derivatives of the master integrals with respect to the invariants of the problem, and then re-express the result in terms of the master integrals themselves. This leads to a system of differential equations that can then be solved once appropriate boundary conditions are found. In our case, we take advantage of the introduction of the auxiliary variable $x$, as shown in Eq. (2.1), and take derivatives with respect to this variable. For example, consider the following two master integrals, which were needed to obtain $A_{Q q}^{(3), P S}$,

$$
\begin{aligned}
& M_{1}(x)=\int \frac{d^{D} k_{1}}{(2 \pi)^{D}} \frac{d^{D} k_{2}}{(2 \pi)^{D}} \frac{d^{D} k_{3}}{(2 \pi)^{D}} \frac{1}{D_{1} D_{2} D_{3} D_{4} D_{5} D_{6} D_{7}}, \\
& M_{2}(x)=\int \frac{d^{D} k_{1}}{(2 \pi)^{D}} \frac{d^{D} k_{2}}{(2 \pi)^{D}} \frac{d^{D} k_{3}}{(2 \pi)^{D}} \frac{1}{D_{1}^{2} D_{2} D_{3} D_{4} D_{5} D_{6} D_{7}},
\end{aligned}
$$

where

$$
\begin{gathered}
D_{1}=\left(k_{1}-p\right)^{2}, \quad D_{2}=\left(k_{2}-p\right)^{2}, \quad D_{3}=k_{3}^{2}-m^{2}, \quad D_{4}=\left(k_{1}-k_{3}\right)^{2}-m^{2} \\
D_{5}=\left(k_{2}-k_{3}\right)^{2}-m^{2}, \quad D_{6}=1-x \Delta . k_{3}, \quad D_{7}=1-x\left(\Delta \cdot k_{3}-\Delta \cdot k_{1}\right) .
\end{gathered}
$$


Here $m$ is the mass of the heavy quark, and $p$ the momentum of the external light quark, which is taken on-shell $\left(p^{2}=0\right)$. Taking derivatives with respect to $x$ we obtain

$$
\begin{aligned}
\frac{d}{d x} M_{1}(x)= & \frac{1}{1-x}\left(2+\varepsilon-\frac{1}{x}\right) M_{1}(x)+\frac{2 x}{1-x} M_{2}(x)+\frac{K_{1}(x)}{1-x} \\
\frac{d}{d x} M_{2}(x)= & -\frac{1}{1-x}\left(\frac{1-2 \varepsilon}{x}+\frac{3}{2} \varepsilon-2\right) M_{2}(x) \\
& +\frac{\varepsilon}{4}(2+3 \varepsilon) \frac{1}{1-x}\left(\frac{1}{x^{2}}-\frac{1}{x}\right) M_{1}(x)+\frac{K_{2}(x)}{1-x}
\end{aligned}
$$

where $K_{1}(x)$ and $K_{2}(x)$ are linear combinations of sub-sector master integrals that have been solved previously. In Eqs. $(2.7,2.8)$, we have set the mass $m$ and $\Delta . p$ to 1 for simplicity. Now we undo the introduction of the variable $x$. Since

$$
M_{1}(x) \propto \sum_{N=0}^{\infty} x^{N} F_{1}(N) \quad \text { and } \quad M_{2}(x) \propto \sum_{N=0}^{\infty} x^{N} F_{2}(N)
$$

we obtain the following system of difference equations,

$$
\begin{aligned}
(N+2) F_{1}(N+1)-(N+2+\varepsilon) F_{1}(N)-2 F_{2}(N-1) & =K_{1}(N), \\
(N+2-2 \varepsilon) F_{2}(N+1)-\left(N+2-\frac{3}{2} \varepsilon\right) F_{1}(N) & \\
-\frac{\varepsilon}{4}(2+3 \varepsilon)\left(F_{1}(N+2)-F_{1}(N+1)\right) & =K_{2}(N),
\end{aligned}
$$

where $K_{1}(N)$ and $K_{2}(N)$ are the $N$ th terms of the Taylor expansions of $K_{1}(x)$ and $K_{2}(x)$, respectively. This system can now be solved using Sigma, together with the Mathematica package Oresys [63]; for further details on this approach we refer to [57]. In order to be able to do so, we need to obtain a few initial values for the integrals under consideration, which we can do using the program MATAD [64] or by doing reductions of tensor integrals to scalar integrals [10]. Many of the master integrals needed to obtain $A_{Q q}^{(3), \text { PS }}$ and some of the terms $\propto T_{F}^{2}$ in $A_{g g}^{(3)}$ were calculated this way. Recently, 3-loop quarkonic ladder and $V$-topology diagrams have also been obtained using this method, cf. [57].

\section{Results}

The expressions obtained for the operator matrix elements $A_{g q}^{(3)}, A_{q q}^{(3), \mathrm{NS}}$ and $A_{q q}^{(3), \mathrm{TR}}$ have been found to be given in terms of harmonic sums [65,66] of up to weight five. For $A_{Q q}^{(3), P S}$ for the first time generalized sums $[44,67]$ appear in the final answer, namely,

$$
S_{a, \vec{b}}(\zeta, \vec{\xi} ; N)=\sum_{k=1}^{N} \frac{\zeta^{k}}{k^{a}} S_{\vec{b}}(\vec{\xi} ; k) \equiv S_{a, \vec{b}}(\zeta, \vec{\xi})
$$

In particular, the constant term in $A_{Q q}^{(3), P S}$ contains the following generalized sums

$$
\begin{aligned}
& S_{1}\left(\frac{1}{2}\right), S_{2}\left(\frac{1}{2}\right), S_{3}\left(\frac{1}{2}\right), S_{1,1}\left(\frac{1}{2}, 1\right), S_{1,1}\left(1, \frac{1}{2}\right), S_{2,1}\left(\frac{1}{2}, 1\right), S_{1,2}\left(\frac{1}{2}, 1\right), S_{2,1}\left(1, \frac{1}{2}\right) \\
& S_{1,2}\left(1, \frac{1}{2}\right), S_{1,1,1}\left(\frac{1}{2}, 1,1\right), S_{1,1,1}\left(1, \frac{1}{2}, 1\right), S_{1,1,1}\left(1,1, \frac{1}{2}\right), S_{3}(2), S_{1,2}(2,1), S_{2,1}(2,1),
\end{aligned}
$$


etc., where we have omitted the explicit dependence on $N$.

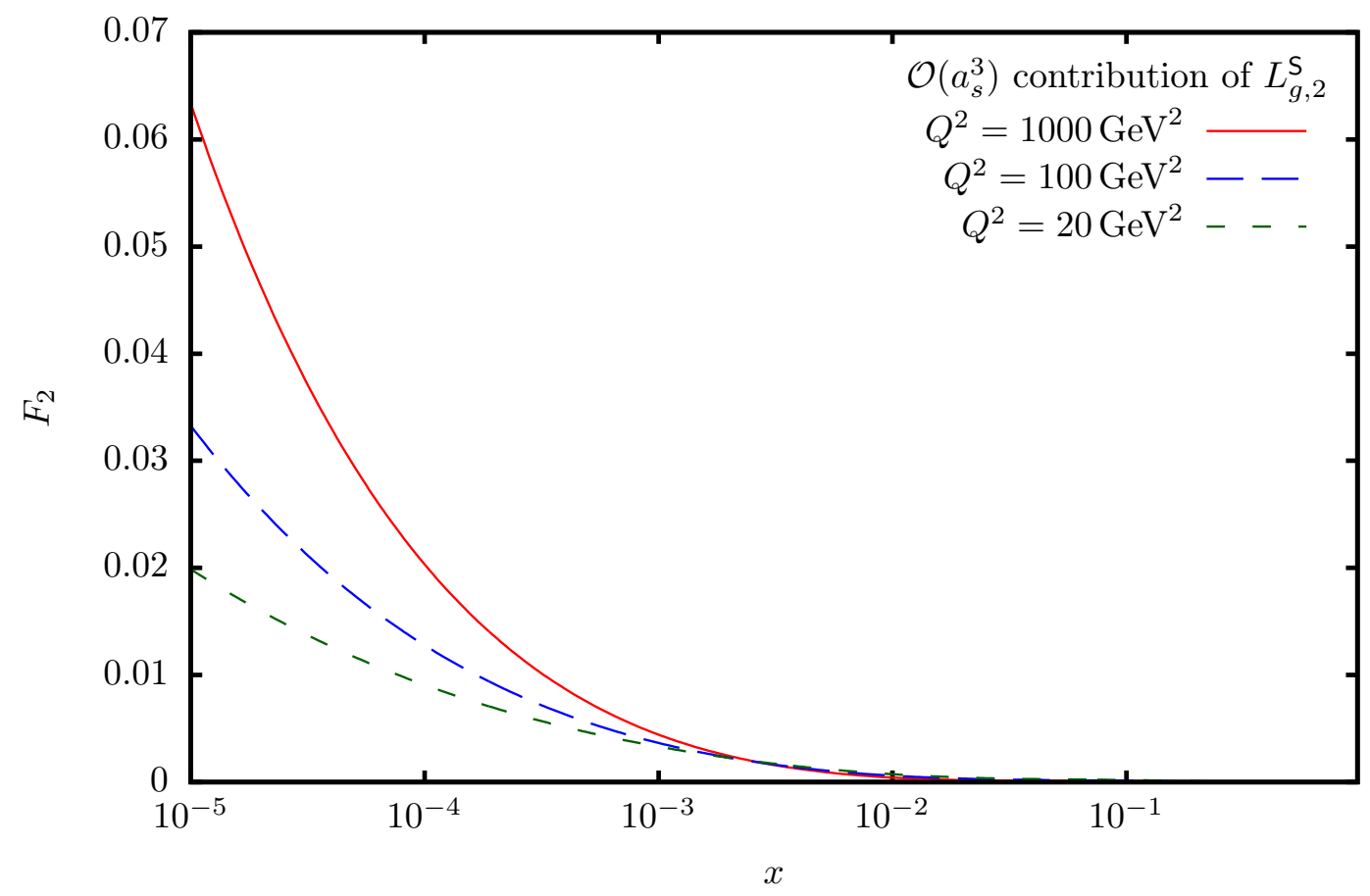

Figure 1: The $O\left(a_{s}^{3}\right)$ contribution by $L_{g, 2}^{\mathrm{S}}$ to the structure function $F_{2}\left(x, Q^{2}\right)$ for $m_{c}=1.59 \mathrm{GeV}$ using the parton distributions [15] (from Ref. [8]).

In terms of a Mellin transform,

$$
\hat{f}(N)=\int_{0}^{1} d x x^{N-1} f(x)
$$

these sums lead to generalized harmonic polylogarithms [44]. Using a recent reduction mechanism available in HarmonicSums we were able to transform the physical result into the harmonic polylogarithms [68] evaluated at $x$ and $1-2 x$. There are also other equivalent representations requiring generalizations of the Mellin transform, cf. [7].

In the case of the terms $\propto T_{F}^{2}$ in $A_{g g}^{(3)}$ [10] and for $V$-graph topologies contributing to $A_{Q g}^{(3)}$ [55] we also found finite nested (inverse) binomial sums over (generalized) harmonic sums such as

$$
\frac{1}{4^{N}}\left(\begin{array}{c}
2 N \\
N
\end{array}\right) \sum_{k=0}^{N} \frac{4^{k}}{k^{l}\left(\begin{array}{c}
2 k \\
k
\end{array}\right)} S_{1}(k), \quad l \in \mathbb{N}
$$

or

$$
\sum_{i=1}^{N}\left(\begin{array}{c}
2 i \\
i
\end{array}\right)(-2)^{i} \sum_{j=1}^{i} \frac{1}{j\left(\begin{array}{c}
(j) \\
j
\end{array}\right)} S_{1,2}\left(\frac{1}{2},-1 ; j\right),
$$

where $S_{\vec{a}}(N)$ denotes a nested harmonic sum.

Doing the inverse Mellin transform of these sums we find that these are expressed in terms of iterated integrals over root-valued alphabets. In total, we have found that 33 new letters are needed in the algebraically irreducible representations for the calculations we have done so far. 
The calculation of all these OMEs has allowed us also to check the corresponding contributions to the 3-loop anomalous dimensions. We find perfect agreement with the literature. In the case of transversity, these have been calculated for the first time ab initio.

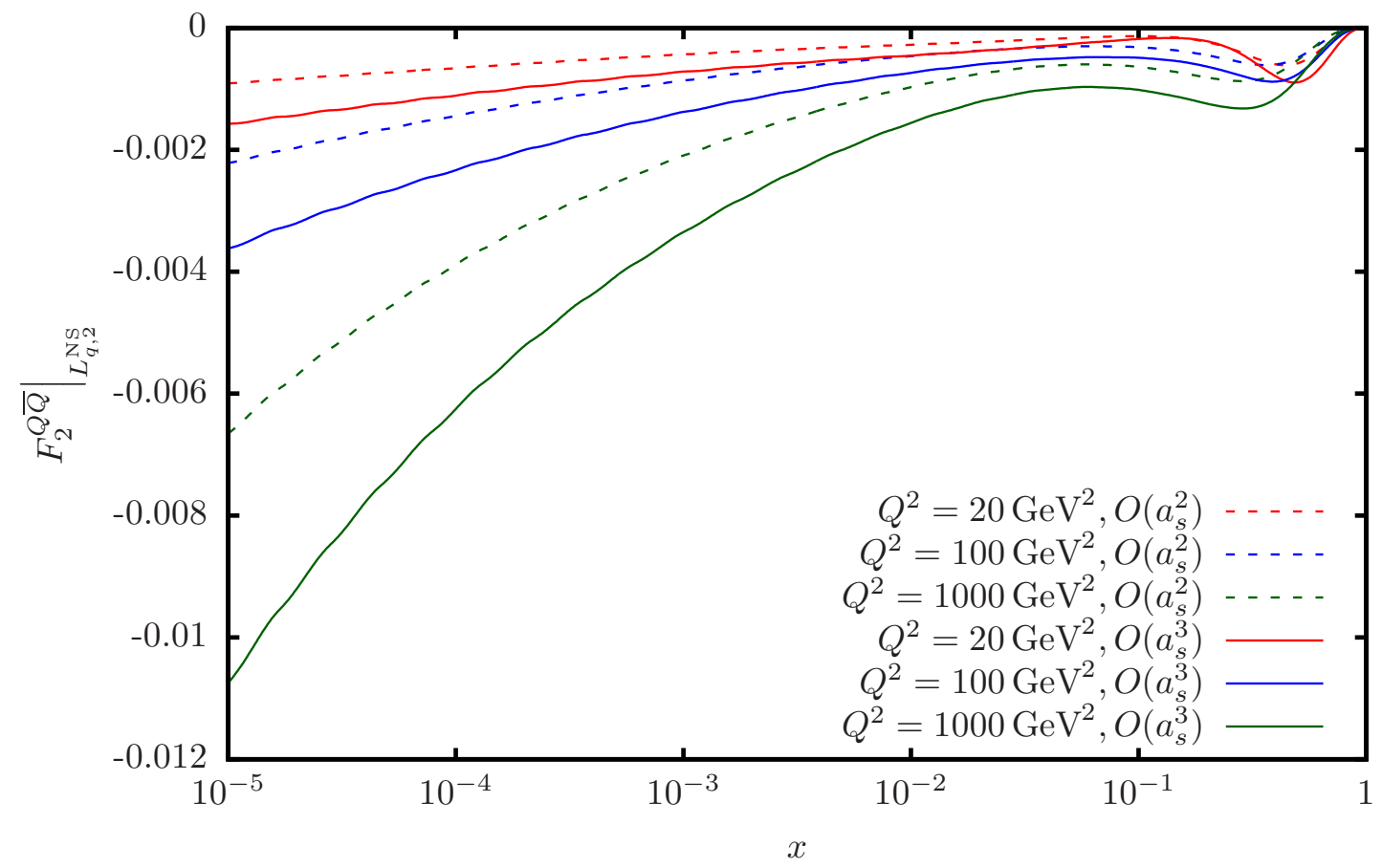

Figure 2: The flavor non-singlet contribution of the Wilson coefficient $L_{q, 2}^{\mathrm{NS}}$ to the structure function $F_{2}\left(x, Q^{2}\right)$ at 2- and 3-loop order using the NNLO parton distribution functions [15] in the on-shell scheme for $m_{c}=$ $1.59 \mathrm{GeV}$ (from Ref. [6]).

Having calculated these OMEs, the remaining tasks are the convolution with the massless Wilson coefficients and then with the PDFs, in order to obtain the contributions to the structure functions. We have obtained numerical results for the Wilson coefficients $L_{q,(2)}^{\mathrm{PS}}, L_{g,(2)}^{\mathrm{S}}[8]$ and $L_{q,(2)}^{\mathrm{NS}}$ [6] to 3-loop order. In Figure 1 the 3-loop corrections by the Wilson coefficient $L_{g, 2}^{S}$ is shown. In the kinematic region probed by HERA it reaches $\sim 1 \%$, i.e. the experimental accuracy and is therefore of importance. They are larger than the 2-loop corrections for this quantity, due to a term $\propto 1 / z$ emerging first in the 3-loop corrections, cf. [8]. In Figure 2, we show the contribution of the heavy flavor non-singlet Wilson coefficient to structure function $F_{2}\left(x, Q^{2}\right)$ at 2- and 3-loop order, for different values of $Q^{2}$. They turn out to be smaller than $1 \%$ in the kinematic region of HERA. In Ref. [6] we also presented the complete transformation coefficients in the VFNS in the non-singlet case at 3-loop order. Future high-luminosity machines such as the EIC [69] will reach a much higher resolution for $F_{2}\left(x, Q^{2}\right)$ than HERA. Here all these terms will be of experimental relevance. Numerical results on the pure-singlet contributions will be given later this year.

\section{Conclusions}

Considerable progress has been made recently in the calculation of the NNLO heavy flavor contri- 
butions to the structure functions in DIS for large values of $Q^{2}$. By now, six out of eight operator matrix elements (and the associated anomalous dimensions) have been completed, and partial results are available for the remaining two OMEs. This progress was possible thanks to the development of new computer algebra and mathematical technologies. Several programs have played a crucial role in these calculations, such as Reduze2 for the reduction to master integrals, and Sigma, HarmonicSums, EvaluateMultisums, SumProduction and OreSys for summation algorithms and the solution of difference equations. These programs and the algorithms associated with them continue to be developed and refined as we encounter ever more challenging problems in this endeavor. The 3-loop heavy flavor Wilson coefficients calculated so far yield contributions to $F_{2}\left(x, Q^{2}\right)$ of $O(\lesssim 1 \%)$, cf. [6,8], reaching the experimental accuracy of the structure function $F_{2}\left(x, Q^{2}\right)$ at HERA. We will report on numerical results for further Wilson coefficients and OMEs in the future. The completion of this project is underway and will allow us to make more precise determinations of $\alpha_{s}$ and $m_{c}$, the parton distribution functions, as well as to establish the VFNS at NNLO, needed for predictions at hadron colliders such as the LHC.

\section{References}

[1] M. Buza, Y. Matiounine, J. Smith, R. Migneron and W. L. van Neerven, Nucl. Phys. B 472 (1996) 611 [hep-ph/9601302].

[2] I. Bierenbaum, J. Blümlein and S. Klein, Nucl. Phys. B 820 (2009) 417 [arXiv:0904.3563 [hep-ph]].

[3] J.A.M. Vermaseren, A. Vogt and S. Moch, Nucl. Phys. B 724 (2005) 3 [hep-ph/0504242].

[4] J. Ablinger, J. Blümlein, S. Klein, C. Schneider and F. Wißbrock, Nucl. Phys. B 844 (2011) 26 [arXiv:1008.3347 [hep-ph]].

[5] J. Ablinger, J. Blümlein, A. De Freitas, A. Hasselhuhn, A. von Manteuffel, M. Round, C. Schneider and F. Wißbrock, Nucl. Phys. B 882 (2014) 263 [arXiv:1402.0359 [hep-ph]].

[6] J. Ablinger, A. Behring, J. Blümlein, A. De Freitas, A. Hasselhuhn, A. von Manteuffel, M. Round, C. Schneider, and F. Wißbrock, arXiv:1406.4654 [hep-ph], Nucl. Phys. B (2014) in press.

[7] J. Ablinger et al., DESY 13-232.

[8] A. Behring, I. Bierenbaum, J. Blümlein, A. De Freitas, S. Klein and F. Wißbrock, arXiv:1403.6356 [hep-ph].

[9] J. Blümlein, A. De Freitas, W. L. van Neerven and S. Klein, Nucl. Phys. B 755 (2006) 272 [hep-ph/0608024].

[10] J. Ablinger, J. Blümlein, A. De Freitas, A. Hasselhuhn, A. von Manteuffel, M. Round and C. Schneider, Nucl. Phys. B 885 (2014) 280 [arXiv:1405.4259 [hep-ph]].

[11] J. Blümlein, A. Hasselhuhn, S. Klein and C. Schneider, Nucl. Phys. B 866 (2013) 196 [arXiv:1205.4184 [hep-ph]].

[12] A. Behring, J. Blümlein, A. De Freitas, T. Pfoh, C. Raab, M. Round, J. Ablinger, A. Hasselhuhn, C. Schneider, and F. Wißbrock. PoS RADCOR 2013 (2014) 058 [arXiv:1312.0124 [hep-ph]].

[13] S. Alekhin, J. Blümlein, K. Daum, K. Lipka and S. Moch, Phys. Lett. B 720 (2013) 172 [arXiv:1212.2355 [hep-ph]]. 
[14] P. Jimenez-Delgado and E. Reya, Phys. Rev. D 89 (2014) 074049 [arXiv:1403.1852 [hep-ph]]; R. S. Thorne, arXiv:1402.3536 [hep-ph];

R. D. Ball et al. [NNPDF Collaboration], Nucl. Phys. B 877 (2013) 290 [arXiv:1308.0598 [hep-ph]]; J. Gao, M. Guzzi, J. Huston, H. -L. Lai, Z. Li, P. Nadolsky, J. Pumplin and D. Stump et al., Phys. Rev. D 89 (2014) 033009 [arXiv:1302.6246 [hep-ph]].

[15] S. Alekhin, J. Blümlein and S. Moch, Phys. Rev. D 89 (2014) 054028 [arXiv:1310.3059 [hep-ph]].

[16] M. Buza, Y. Matiounine, J. Smith and W. L. van Neerven, Eur. Phys. J. C 1 (1998) 301 [hep-ph/9612398].

[17] J. Blümlein and F. Wißbrock, DESY 14-019.

[18] J. Ablinger, J. Blümlein, S. Klein, C. Schneider and F. Wißbrock, arXiv:1106.5937 [hep-ph].

[19] J. Ablinger, J. Blümlein, A. Hasselhuhn, S. Klein, C. Schneider and F. Wißbrock, PoS RADCOR2011 (2011) 031 [arXiv:1202.2700 [hep-ph]].

[20] J. Ablinger, J. Blümlein, A. De Freitas, A. Hasselhuhn, A. von Manteuffel, M. Round, C. Schneider and F. Wißbrock, arXiv:1407.2821 [hep-ph].

[21] M. Buza and W. L. van Neerven, Nucl. Phys. B 500 (1997) 301 [hep-ph/9702242].

[22] J. Blümlein, A. Hasselhuhn and T. Pfoh, Nucl. Phys. B 881 (2014) 1 [arXiv:1401.4352 [hep-ph]].

[23] S.W.G. Klein, arXiv:0910.3101 [hep-ph].

[24] P. Nogueira, J. Comput. Phys. 105 (1993) 279.

[25] J.A.M. Vermaseren, math-ph/0010025;

M. Tentyukov and J.A.M. Vermaseren, Comput. Phys. Commun. 181 (2010) 1419 [hep-ph/0702279].

[26] A. von Manteuffel and C. Studerus, arXiv:1201.4330 [hep-ph];

C. Studerus, Comput. Phys. Commun. 181 (2010) 1293 [arXiv:0912.2546 [physics.comp-ph]].

[27] R.H. Lewis, Computer Algebra System Fermat, http://home.bway . net/lewis.

[28] C. W. Bauer, A. Frink and R. Kreckel, Symbolic Computation 33 (2002) 1, cs/0004015 [cs-sc].

[29] S. Laporta, Int. J. Mod. Phys. A 15 (2000) 5087 [hep-ph/0102033].

[30] C. Schneider, Sém. Lothar. Combin. 56 (2007) 1, article B56b.

[31] C. Schneider, Computer Algebra in Quantum Field Theory: Integration, Summation and Special Functions Texts and Monographs in Symbolic Computation eds. C. Schneider and J. Blümlein (Springer, Wien, 2013) 325 arXiv:1304.4134 [cs.SC].

[32] M. Karr 1981 J. ACM 28 (1981) 305.

[33] C. Schneider, Symbolic Summation in Difference Fields Ph.D. Thesis RISC, Johannes Kepler University, Linz technical report 01-17 (2001).

[34] C. Schneider, J. Differ. Equations Appl. 11 (2005) 799.

[35] C. Schneider, J. Algebra Appl. 6 (2007) 415.

[36] C. Schneider, J. Symbolic Comput. 43 (2008) 611 [arXiv:0808.2543].

[37] C. Schneider, Appl. Algebra Engrg. Comm. Comput. 21 (2010) 1. 
[38] C. Schneider, Motives, Quantum Field Theory, and Pseudodifferential Operators (Clay Mathematics Proceedings Vol. 12, eds. A. Carey, D. Ellwood, S. Paycha and S. Rosenberg (Amer. Math. Soc) (2010), 285 arXiv:0808.2543.

[39] C. Schneider, Ann. Comb. 14 (2010) 533 [arXiv:0808.2596].

[40] C. Schneider, in : Lecture Notes in Computer Science (LNCS) eds. J. Guitierrez, J. Schicho, M. Weimann, in press, arXiv:1307.7887 [cs.SC] (2013).

[41] J. Ablinger, arXiv:1011.1176 [math-ph].

[42] J. Ablinger, arXiv:1305.0687 [math-ph].

[43] J. Ablinger, J. Blümlein and C. Schneider, J. Math. Phys. 52 (2011) 102301 [arXiv:1105.6063 [math-ph]] and in preparation.

[44] J. Ablinger, J. Blümlein and C. Schneider, J. Math. Phys. 54 (2013) 082301 [arXiv:1302.0378 [math-ph]].

[45] J. Ablinger, J. Blümlein, S. Klein and C. Schneider, Nucl. Phys. Proc. Suppl. 205-206 (2010) 110 [arXiv:1006.4797 [math-ph]];

J. Blümlein, A. Hasselhuhn and C. Schneider, PoS RADCOR 2011 (2011) 032 [arXiv:1202.4303 [math-ph]];

[46] C. Schneider, J. Phys. Conf. Ser. 523 (2014) 012037 [arXiv:1310.0160 [cs.SC]].

[47] W.N. Bailey, Generalized Hypergeometric Series, (Cambridge University Press, Cambridge, 1935); L.J. Slater, Generalized Hypergeometric Functions, (Cambridge University Press, Cambridge, 1966); P. Appell and J. Kampé de Fériet, Fonctions Hypergéométriques et Hyperspériques, Polynomes D' Hermite, (Gauthier-Villars, Paris, 1926);

P. Appell, Les Fonctions Hypergëométriques de Plusieur Variables, (Gauthier-Villars, Paris, 1925);

J. Kampé de Fériet, La fonction hypergëométrique,(Gauthier-Villars, Paris, 1937);

H. Exton, Multiple Hypergeometric Functions and Applications, (Ellis Horwood, Chichester, 1976);

H. Exton, Handbook of Hypergeometric Integrals, (Ellis Horwood, Chichester, 1978);

H.M. Srivastava and P.W. Karlsson, Multiple Gaussian Hypergeometric Series, (Ellis Horwood, Chicester, 1985).

[48] I. Bierenbaum, J. Blümlein and S. Klein, Nucl. Phys. B 780 (2007) 40 [hep-ph/0703285];

I. Bierenbaum, J. Blümlein, S. Klein and C. Schneider, Nucl. Phys. B 803 (2008) 1 [arXiv:0803.0273 [hep-ph]];

I. Bierenbaum, J. Blümlein and S. Klein, Phys. Lett. B 672 (2009) 401 [arXiv:0901.0669 [hep-ph]].

[49] J. Ablinger, J. Blümlein, A. Hasselhuhn, S. Klein, C. Schneider and F. Wißbrock, Nucl. Phys. B 864 (2012) 52 [arXiv:1206.2252 [hep-ph]].

[50] H. Mellin, Acta Societatis Scientiarum Fennicae, XX.(7) (1895) 1; Math. Ann. 68 (1910) 305.

[51] E.W. Barnes, Proc. Lond. Math. Soc. (2) 6 (1908) 141; Quart. J. Math. 41 (1910) 136.

[52] V. A. Smirnov, Phys. Lett. B 460 (1999) 397 [hep-ph/9905323].

[53] V. A. Smirnov, Feynman Integral Calculus, (Springer, Berlin, 2006).

[54] F. Brown, Commun. Math. Phys. 287 (2009) 925 [arXiv:0804.1660 [math.AG]].

[55] J. Ablinger, J. Blümlein, C. Raab, C. Schneider and F. Wißbrock, Nucl. Phys. B885 (2014) 409 arXiv:1403.1137 [hep-ph]. 
[56] A. V. Kotikov, Phys. Lett. B 254 (1991) 158;

M. Caffo, H. Czyz, S. Laporta and E. Remiddi, Acta Phys. Polon. B 29 (1998) 2627 [hep-th/9807119]; Nuovo Cim. A 111 (1998) 365 [hep-th/9805118];

T. Gehrmann and E. Remiddi, Nucl. Phys. B 580 (2000) 485 [hep-ph/9912329];

M. Caffo, H. Czyz and E. Remiddi, Nucl. Phys. B 634 (2002) 309 [hep-ph/0203256].

[57] J. Blümlein, A. De Freitas and C. Schneider, arXiv:1407.2537 [cs.SC], PoS (LL2014) 017.

[58] J. Ablinger, PoS (LL2014) 019.

[59] J. Ablinger, J. Blümlein, C. Raab, and C. Schneider, PoS (LL2014) 020.

[60] J. Ablinger, J. Blümlein, C. G. Raab and C. Schneider, arXiv:1407.1822 [hep-th].

[61] J. Ablinger and J. Blümlein, arXiv:1304.7071 [math-ph].

[62] M. Czakon, Comput. Phys. Commun. 175 (2006) 559 [hep-ph/0511200].

[63] S. Gerhold, Uncoupling systems of linear ore operator equations, Master's thesis, RISC, J. Kepler University, Linz, 2002.

[64] M. Steinhauser, Comput. Phys. Commun. 134 (2001) 335 [hep-ph/0009029].

[65] J.A.M. Vermaseren, Int. J. Mod. Phys. A 14 (1999) 2037 [arXiv:hep-ph/9806280].

[66] J. Blümlein and S. Kurth, Phys. Rev. D 60 (1999) 014018 [arXiv:hep-ph/9810241].

[67] S. Moch, P. Uwer and S. Weinzierl, J. Math. Phys. 43 (2002) 3363 [hep-ph/0110083].

[68] E. Remiddi and J. A. M. Vermaseren, Int. J. Mod. Phys. A 15 (2000) 725 [hep-ph/9905237].

[69] D. Boer, M. Diehl, R. Milner, R. Venugopalan, W. Vogelsang, D. Kaplan, H. Montgomery and S. Vigdor et al., arXiv:1108.1713 [nucl-th];

A. Accardi, J. L. Albacete, M. Anselmino, N. Armesto, E. C. Aschenauer, A. Bacchetta, D. Boer and W. Brooks et al., arXiv:1212.1701 [nucl-ex]. 